Romero Gómez, Pepe.

Profesor Titular de Universidad, Universitat Politècnica de València, Departamento de Escultura.

Garriga Inarejos, Rocío.

Profesora Ayudante Doctora, Universitat Politècnica de València, Departamento de Escultura.

\title{
Puntos de encuentro entre las fallas experimentales y el arte contemporáneo
}

\section{Meeting points between experimental "fallas" and contemporary art}

TIPO DE TRABAJO: Comunicación.

PALABRAS CLAVE

Arte contemporáneo, fallas, experimentación, innovación, investigación, escultura.

KEY WORDS

Contemporary art, "fallas", experimentation, innovation, research, sculpture.

RESUMEN

Desde el Proyecto I+D Aportaciones y apropiaciones mutuas entre las Fallas Experimentales de la ciudad de Valencia y el Arte Contemporáneo, 1998-2018; así como desde la Unidad Mixta de Investigación EFIMERE, que reúne investigadores/as de la UPV y de la UV, estamos elaborando un estudio con el objetivo de reflexionar y hacer visibles los puntos de encuentro existentes entre las fallas experimentales de la ciudad de Valencia y el arte contemporáneo.

Con el fin de esclarecer tales conexiones hemos llevado a cabo una serie de entrevistas a personas que participan en las fallas experimentales. La primera cuestión que abordaremos es la problemática relacionada con lo que se entiende por Fallas I+E (Innovación y Experimentación), dado que su definición es vaga y no existe consenso al respecto. Después, tomando como eje el análisis de ese trabajo de campo, así como el estudio de otros documentos, entre ellos Llibrets, tesis doctorales específicas y otras publicaciones históricas relacionadas, reflexionaremos sobre las formas que presentan los monumentos según los materiales, las técnicas, las variables compositivas empleadas y la relación monumento-espacio público así como los motivos críticos que llevan a tomar tales decisiones. Finalmente iremos más allá del monumento y abordaremos los modos en que las comisiones de las Fallas I+E se relacionan con sus creadores: revisando y actualizando las tradiciones de la fiesta, del uso del espacio público y del resto de actividades culturales que en este contexto se generan.

El estudio de estas cuestiones pondrá de manifiesto la naturaleza de las relaciones que se dan entre las Fallas I+E y otras propuestas específicas de arte contemporáneo.

\section{ABSTRACT}

From the $R+D$ Project Contributions and mutual appropriations between the Experimental "Fallas" of the city of Valencia and Contemporary Art, 1998-2018; as well as from the EFIMERE Joint Research Unit, which brings together researchers from the UPV and the UV, we are preparing a study with the aim of reflecting and making visible the existing meeting points between the experimental "fallas" of the city of Valencia and contemporary art.

In order to clarify such connections we have conducted a series of interviews with people who participate in the experimental "fallas". The first issue we will address is the problem related to what is understood by I + E Fallas (Innovation and Experimentation), given that 
its definition is vague and there is no consensus on this. Then, taking as an axis the analysis of this field work, as well as the study of other documents, among them Llibrets, specific Doctoral Theses and other related historical publications, we will reflect on the forms presented by the monuments according to materials, techniques, compositional variables used and the monument-public space relationship as well as the critical reasons that lead to such decisions. Finally we will go beyond the monument and approach the ways in which the commissions of the Fallas I + E are related to their creators: reviewing and updating the traditions of the party, the use of public space and the rest of cultural activities that in this context they generate.

The study of these issues will highlight the nature of the relationships that occur between the I + E Fallas and other specific proposals of contemporary art.

\section{INTRODUCCIÓN}

En 1991 el IVAJ (Institut Valencià de la Joventut) creó los premios a las fallas experimentales con el fin de impulsar la renovación formal de los monumentos falleros tradicionales. Estos últimos se caracterizan por tener una estructura piramidal en cuya base se extienden las distintas escenas; por las formas redondeadas de unos ninots - caricatura, similares a los dibujos de la animación 3D; por los acabados pictóricos que presentan, con tonos desaturados que siempre se aplican enfatizando los volúmenes de las formas; $y$ por el tratamiento espacial que presentan, porque los lugares donde se emplazan estas fallas se acotan con vallas para proteger su seguridad o con fines lucrativos, dependiendo de la sección a la que pertenezcan.

Los monumentos tradicionales falleros también presentan desafíos, su envergadura es uno de los más importantes, pues contienen multitud de figuras ornamentales y a veces arriesgadas estructuras, confiriéndoles su aspecto barroco característico. Sin embargo, las constantes mencionadas homogeneizan las propuestas confiriéndoles un aspecto similar y kitsch que se generaliza entre las comisiones falleras más tradicionales que entienden la definición del monumento según estas características. Una definición estética apoyada por los Premios de la Junta Central Fallera. El exceso ornamental y la crítica meliflua (o su total ausencia) de la mayor parte de las fallas de Valencia contrasta con las fallas experimentales, unos pocos monumentos más sobrios, en algunos casos claramente minimalistas, cuyos lemas están relacionados con los problemas sociales actuales cuestionando, a su vez, el significado de cada uno de los componentes de la fiesta.

En 1998 el Ayuntamiento de Valencia instaura los Premios a las Fallas Innovadoras para respaldar las propuestas de un grupo de comisiones que defendía, junto a los artistas que contrataba o en sus creaciones colectivas, una manera de hacer distinta a la de la mayoría de las fallas. El reconocimiento a este tipo de creaciones, que presentan preocupaciones no solo estéticas sino también discursivas, comienza a consolidarse, y a día de hoy cabe reconocer en ellos también una serie de características que los definen. No obstante, a diferencia de lo que sucede con los monumentos tradicionales falleros, en el caso de las fallas I+E, los desafíos que se abordan se resisten a la clasificación y las características que las definen son dinámicas, mudables según los principios de innovación y experimentación que rigen su desarrollo.

De acuerdo con lo anterior, y siguiendo las creaciones experimentales que se han venido haciendo desde el año 1991, pueden observarse algunos rasgos generales o comunes a la mayor parte de todas ellas. Las fallas innovadoras suelen realizarse con materiales no contaminantes y de combustión más limpia (es uno de los criterios para la concesión de los premios de esta sección); plantean una relación más compleja y diversa con el espacio en el que se plantan, recuperan la pared del edificio como soporte o desarrollan su narración en espacios concebidos a modo de instalación de arte contemporáneo... En algunos casos los monumentos se convierten en estructuras geométricas minimalistas, e incorporan el uso de tecnologías tales como el vídeo-mapping o elementos sonoros. Son varios los casos de fallas experimentales en los que se observa un tratamiento íntegro de los sentidos perceptivos, sumando a la vista y el oído, el olfato, el tacto e incluso el gusto. Cabe señalar, además, que en este tipo de fallas se someten a revisión algunos de los ejes que sustentan las creaciones tradicionales, como sucede, por ejemplo, con la idea tácita de que los ninots deban ser antropomórficos; además, en las fallas experimentales estos últimos ya no se ubican rodeando un eje central, dejan de someterse a esa estructura y se esparcen en el espacio; un espacio que por otra parte, acotado o no con vallas perimetrales (que en la mayoría de las ocasiones se usan únicamente por motivos de seguridad durante la noche), termina siendo de un modo u otro, transitable, accesible al público general sustituyendo los fines lucrativos (cobrar entrada para acceder al interior de la falla) por fines de carácter comunitario y participativo.

\section{METODOLOGÍA}

Con el fin de esclarecer las conexiones que se dan entre las fallas I+E y el arte contemporáneo, hemos llevado a cabo una serie de entrevistas a personas que participan o que han tratado teóricamente sobre las fallas experimentales. El análisis de ese trabajo de 
campo, que ha incluido visitas a numerosos talleres que centran su labor en la creación de este tipo de fallas, así como el estudio de otros documentos, entre ellos Llibrets, tesis doctorales específicas y otras publicaciones históricas relacionadas, nos ha servido para reflexionar sobre las formas que presentan los monumentos según los materiales, las técnicas, las variables compositivas empleadas y la relación monumento-espacio público así como los motivos críticos y discursivos que llevan a tomar tales decisiones. Este proceso de investigación ha cristalizado en dos exposiciones que dan a conocer la trayectoria de las Fallas I+E. El título que aglutina ambas muestras es Obrint Portes. Falles Innovadores a la ciutat de Valencia. Ambas se inauguraron la primera semana del mes de marzo de 2019, una de ellas en la Sala de Exposiciones del Edificio del Rectorado de la Universitat Politècnica de Valencia; la otra, en la Sala de Exposiciones Josep Renau de la Facultat de Belles Arts de esta misma Universidad.

En la exposición ubicada en el Edificio del Rectorado se ha presentado un vídeo dividido en cuatro secciones y cada una de ellas cuenta con una hora de duración aproximadamente. La primera de ellas, titulada Sociologia i Comunicació, presenta entrevistas realizadas a académicos, investigadores o teóricos del mundo de las fallas: Gil Manuel Hernández nos habla de las fallas como objeto de discusión e investigación científica y artística; Enrique Collado de las fallas como elemento de comunicación; y Antonio Ariño se centra en el valor sociológico de las fallas como elemento generador de identidad y comunidad. El segundo y el tercer capítulos de este vídeo se centran en la Història de las fallas I+E hasta su momento actual. En ambas secciones participan artistas falleros o creadores de fallas experimentales que iniciaron y han continuado llevando a cabo cambios formales con sus propuestas innovadoras. Las personas entrevistadas que integran estas dos partes del audiovisual son: Pilar y María José Luna (hijas de Vicente Luna), Alfredo Ruiz, Manolo Martín, Óscar Mora, Miguel Arraiz, Ibán Ramón, Anna Ruiz y Giovanni Nardin, José Nicola. El cuarto capítulo Crítica Integral $i$ Alternatives, muestra el trabajo de aquellas comisiones en las que las creaciones se llevan a cabo de forma colaborativa, como es el caso de la Falla Borrull-Socors; o de las comisiones que van todavía más allá y que plantean una crítica integral a la jerarquizada fiesta fallera y a sus manifestaciones religiosas, los casos de la Falla Arrancapins y de FAPOCO (Falles Populars i Combatives). Finalmente, el vídeo que se muestra en Sala de Exposiciones Josep Renau comienza con una reflexión sobre la trayectoria de la Falla UPV de Salomé Cuesta (Directora del Departamento de Escultura) y José Galindo (Decano de la Facultat de Belles Arts UPV), para dar paso a la concreción de su historia de la mano de Jaume Chornet y Leo Gómez Haro, creadores de la falla.

Presentar este trabajo al público ha exigido un esfuerzo de síntesis en lo referente al material documental obtenido durante la grabación de las entrevistas, material del que nos servimos también para la elaboración del presente texto.

\section{DESARROLLO}

\section{Antecedentes}

Tras el monumento fallero diseñado por Dalí en 1954, que se alejaba de los cánones de la época, podemos considerar a Ricardo Rubert como el artista fallero más innovador de la siguiente década, un autor que se destacó por la realización de monumentos más esquemáticos y estilizados, donde el vacío cobraba gran importancia en los volúmenes de sus formas geométricas. Posteriormente, Vicente Luna, planta en el año 1981 Concorde... Concordia, una falla de escasa altura en la que predomina el eje horizontal sobre el vertical, aspecto siempre criticado por ofrecer una cremá menos impactante. Este mismo creador inicia en el año 1982 un proceso de deconstrucción del monumento, mostrándolo en una fase previa a su acabado. Así, el Atlante Moderno, fue la primera falla que se presentó en "vareta" (tiras de madera que cubren el esqueleto), la primera ocasión en que se mostró el trabajo de ingeniería que suponía la ejecución de esta clase de monumentos. El riesgo que asumió Vicente Luna al presentar un trabajo que muchos criticaron como "inacabado" abrió las puertas a una forma de hacer distinta, una forma de hacer que vería su continuidad en los hermanos Ferrer y en Manolo García.

A partir de entonces la falla municipal llevaría el sello de Manolo Martín, un artista renovador que ha colaborado con multitud de artistas y creadores convirtiendo en volúmenes sus ideas. Comienza en 1985 con el Castillo Disney, y en 1986 y 1987 trabaja a partir de los diseños de Sento Llobell y planta unas fallas en las que colaboran Francis Montesinos y Manuel Vicent. El año 1988 lo hace con Ortifus y posteriormente planta con la comisión de la falla Na Jordana, Mutaciones. Pinotxada Universal, una falla histórica diseñada por Sigfrido Martín Begué, también La Transició, monumento creado junto a Chema Cobo.

Después de las propuestas ejecutadas por Manolo Martín, la falla municipal no recuperará su carácter innovador hasta el año 2014. En aquel momento, asume el encargo Manolo García, que recupera la vareta y hace de ella su señal de identidad aunque este modo de expresión tuviera otros antecedentes como el mencionado Vicente Luna o los hermanos Ferrer, que en 1997 plantaron La nostra historia. En el 2018 y el 2019, Pepe Latorre y Gabriel Sanz realizan los proyectos de Okuda y de los artistas urbanos valencianos Pichiavo (Juan Antonio Sánchez y Álvaro Hernández).

Pero, como cabe suponer, la innovación y la experimentación durante aquellos años no se redujo a la falla municipal. Alfredo Ruiz es uno de los creadores de fallas más destacados en este sentido, desde 1987 comenzó un proceso de síntesis formal. Con la falla QuartPalomar plantó Com aigua lliure (2002), A la naturalesa (2001) y Propostes per l'harmonia (2000), todas ellas abstractas y con una paleta de color muy reducida o monocromática. Estas fallas fueron realizadas tras haber anulado también el color en las fallas 
figurativas plantadas en la década de los años 90 con la misma comisión. Su proceso de abstracción culminaría en el minimalismo más radical, cuando Alfredo Ruiz presentó en 2008 la falla titulada Poesía Horizontal en Mossen Sorell-Corona. Aquella pieza consistió en una "falca per mantenir la porta oberta», expresión, según sus palabras, de una personalidad absolutamente innovadora al margen de lo establecido.

Como indicábamos anteriormente, en el año 1991 el Institut Valencià de la Joventut IVAJ, creó el premio de fallas experimentales y algunos artistas visuales como Ferrán Martín y Óscar Mora, implicados en la creación fallera, recibieron este galardón en algunas ocasiones. Ferran Martín plantó en la Alameda, el año 1992 la falla El Tubérculo y Óscar Mora plantó en la Plaza del Patriarca, Made in China, en el año 1995. Ambos artistas han colaborado juntos en la creación de diversos monumentos para la Plaza de España, siendo Abierto 24 Horas (1998) una de las fallas más relevantes, en la que participaron también otros artistas del colectivo Purgatori.

\section{Fallas I+E: forma y contenido}

La fallas I+E, se caracterizan por ser monumentos relacionados con las propuestas del arte escultórico contemporáneo, tanto en lo que se refiere a su acabado formal como a su relación con el espacio. En algunos casos las propuestas que presentan los creadores de fallas se acercan al concepto "instalación", predominando el eje horizontal sobre el vertical, mientras que en otros se evidencia un minimalismo formal, aunque aquí, con contenidos narrativos específicos que tratan abiertamente los problemas actuales que a todos nos conciernen, desde un punto de vista crítico no exento de ironía.

Algunas de estas fallas incorporan nuevas tecnologías al monumento, y todas ellas, abren sus espacios y son transitables; sus autores requieren un contacto directo con el espectador llegando incluso a interpelar directamente a la audiencia, ofreciéndole una participación directa, apelando su sentido crítico, a su compromiso, pero haciéndolo siempre de una manera lúdica. Esta última cuestión, cambia la forma de la falla y la convierte además en un elemento activo, dinámico, cambiante, frente al estatismo de los monumentos tradicionales.

La Federación I+E está presidida por José Nicola. A día de hoy son estas las fallas que la componen: Canal Navarres-Vall Albaida (Els Somnis), Mocador de Sagunto, Micer Rabasa-Poeta Maragall, Falla Lepanto-Guillem de Castro, Falla Castielfabib-Marques San Juan, Plaza de Jesús, Falla Borrull-Socors, Jesús Morante Borrás-Caminot (La Punta), Ripalda-Beneficiencia-San Ramon (Ribesan), Plaza del Patriarca (Universitat Vella). Por otro lado, existen otras fallas innovadoras que no están integradas en la Federación, pero que proponen monumentos que se alejan totalmente de los estereotipos más generalizados, y en este grupo podemos incluir a la falla de la Universitat Politécnica de València, la falla Vall de Laguard o la Falla Ciudad del Artista Fallero 2019.

La falla de la UPV del año 2000 fue realizada por Gerardo Sigler, Elisa Lozano y Elena Fernández. Ejecutaron una ola de fuego realizada con cañas naturales. La utilización de estos materiales abría un campo experimental sumamente inspirador, relacionado con nuestro entorno y estrechamente ligado a la condición de investigador de los profesores universitarios. En la actualidad Jaume Chornet y Leo Gómez Haro continúan plantando una falla cargada de contenido discursivo, en la que el humor y la sátira se alían con las nuevas tecnologías, y con propuestas que parten de su investigación con nuevas formas y materiales.

\section{Creaciones performáticas, participativas/colaborativas}

La falla Arrancapins y la Falla Borrull-Socors plantan monumentos realizados íntegramente por sus comisiones, ambas tratan problemas actuales y desde la crítica, aluden a cuestiones de género o a problemas relacionados con la más que cuestionable ilegalidad de los seres humanos que se ven forzados a emigrar. La reflexión que plantean estas fallas incluye a la totalidad de las fallas I+E, lo que las diferencia, es el trabajo colectivo de sus comisiones para sacar adelante el monumento, aunque en algunas ocasiones, no solo Arrancapins o Borrull, sino también la falla de la Plaza del Patriarca lo ha construido colectivamente, debido a su carencia presupuestaria.

Por otro lado, la falla Borrull en sus dos últimas ediciones, alude a los orígenes falleros recuperando el espíritu de la "Estoreta Velleta," construyendo sus fallas a partir de los objetos que aportan sus vecinos. Las fallas Arrancapins y Borrull también coinciden al mostrar su desacuerdo con una estructura jerárquica que impone la elección de la fallera mayor, además Arrancapins se define como entidad laica y no participan en la ofrenda implantada durante el Franquismo en el año 1945, tampoco tienen falleros de honor, sustituyen al presidente por la figura de coordinador/a, todas sus decisiones se toman de forma asamblearia y rechazan los premios, que fueron introducidos por la dictadura.

Hay otras fallas, con presupuestos todavía más reducidos y con un carácter más reivindicativo que son oficiosas y optan por ocupar las calles sin pasar por los trámites oficiales. "La Junta Solar Fallera" coordinó las propuestas de distintos colectivos de barrios de Valencia con una filosofía de autogestión y participación. Solar Corona y su falla popular y combativa (Fapoco) que participaban en este colectivo, genera un proceso colaborativo entre los creadores del monumento, los vecinos del barrio y algunas asociaciones como I'Escoleta del Carme, el Colectivo de Mares i Pares de Ciutat Vella y la Asociación Amaltea. Esta falla se plantea como una oportunidad 
para profundizar en el tejido social del barrio, creando encuentros intergeneracionales y haciendo uso de los espacios comunitarios que se encuentran a su alcance. Desde el año 2013 al 2017, Daniel Tomàs Marquina, Ximo Ortega Garrido, y José Juan Martínez Ballester han colaborado en la elaboración de sus monumentos y en las dinámicas que derivan de este modo de entender las fallas. Como dinamizadores de vecinos y colectivos del barrio, han tocado temas como las elecciones, la gentrificación o el desarrollo turístico, desde una perspectiva crítica y social, temas que por otro lado están directamente relacionados con los solares reivindicados en los que se planta la falla. En las dos últimas ediciones Anais Florin ha relevado a los artistas mencionados, y continua con propuestas combativas recordándonos en parte el espíritu de Josep Renau, que entendía estas celebraciones populares como un instrumento de protesta y lucha.

\section{CONCLUSIONES}

Desde la incorporación de artistas falleros formados en el ámbito de las artes visuales (Diseño, Arquitectura y Bellas Artes) se ha ampliado el registro icónico que en años anteriores se mostraba homogéneo y compacto, y hemos pasado de la criticada excepción, a un espacio de creación fallera que incorpora nuevas tecnologías y aplica procesos creativos que discurren paralelos a los del arte contemporáneo.

Las características que definen y diagnostican un crecimiento que se amplía con los años y que muestra así mismo un desarrollo recíproco entre la sensibilidad plástica, la reflexión discursiva y la sociedad en que vivimos son: el concepto de «instalación» en el que la relación objeto-texto-espacio produce monumentos falleros innovadores, desarrollando principalmente el eje horizontal o apoyando el monumento sobre las paredes de los edificios, así como las propuestas tautológicas en donde la representación objetual se produce a escala 1:1 significando con ello otro tipo de relación entre el espectador y el monumento; también la incorporación de experiencias performativo-temporales que contemplan todas las variables del proyecto del monumento fallero (incluyendo al fuego) convierten al mismo en una obra viva, dinámica y variable en sus sentidos; y finalmente, la interactividad con el espectador que se convierte en ocasiones, en co-creador de la falla aumentándose de este modo las variables descritas anteriormente.

Tal y como hemos visto, el diálogo entre las fallas experimentales y el arte contemporáneo no deja de ser prometedor. El hecho de que hayan comenzado a entrelazarse ambos tipos de expresión/comunicación deja la puerta abierta a la constante innovación y revisión de sus contenidos y sus formas.

\section{FUENTES REFERENCIALES}

Ariño, A. (1990). Fiesta y Sociedad en la Valencia contemporánea. Valencia: Ediciones UV.

Collado, E. (2018). Arte, Fuego y tradición. Valencia: Alejandría Ediciones.

Ferriols, J. E., Pagola, M. y Solá, J. J. (2008). Pirotecnia en Valencia: historia, singularidades, modalidades de fuego y sus protagonistas. Valencia: Ajuntament de Valencia. Delegación de Cultura.

Ramón, I. (2017). Materiales, objetos, procesos y analogías. Valencia: Edición Iban Ramón Rodríguez.

VV.AA. (2008). Falles y Art: 40 anys transitant per la frontera. Valencia: Editorial de la UPV.

VV.AA. (2008). La falla: un artefacte tecnològic. Valencia: Editorial de la UPV. 